\title{
Encoding and Retrieval of Artificial Visuoauditory Memory Traces in the Auditory Cortex Requires the Entorhinal Cortex
}

\author{
Xi Chen, ${ }^{1}$ Yiping Guo, ${ }^{1,2}$ Jingyu Feng, ${ }^{1}$ Zhengli Liao, ${ }^{1}$ Xinjian Li, ${ }^{1}$ Haitao Wang, ${ }^{1,2}$ Xiao Li, ${ }^{1}$ and Jufang He ${ }^{1}$ \\ ${ }^{1}$ Laboratory of Applied Neuroscience, Department of Rehabilitation Sciences, The Hong Kong Polytechnic University, Hung Hom, Kowloon, Hong Kong, \\ and ${ }^{2}$ Guangzhou Institute of Biomedicine and Health, Chinese Academy of Sciences, Guangzhou 510530, China
}

\begin{abstract}
Damage to the medial temporal lobe impairs the encoding of new memories and the retrieval of memories acquired immediately before the damage in human. In this study, we demonstrated that artificial visuoauditory memory traces can be established in the rat auditory cortex and that their encoding and retrieval depend on the entorhinal cortex of the medial temporal lobe in the rat. We trained rats to associate a visual stimulus with electrical stimulation of the auditory cortex using a classical conditioning protocol. After conditioning, we examined the associative memory traces electrophysiologically (i.e., visual stimulus-evoked responses of auditory cortical neurons) and behaviorally (i.e., visual stimulus-induced freezing and visual stimulus-guided reward retrieval). The establishment of a visuoauditory memory trace in the auditory cortex, which was detectable by electrophysiological recordings, was achieved over 20 - 30 conditioning trials and was blocked by unilateral, temporary inactivation of the entorhinal cortex. Retrieval of a previously established visuoauditory memory was also affected by unilateral entorhinal cortex inactivation. These findings suggest that the entorhinal cortex is necessary for the encoding and involved in the retrieval of artificial visuoauditory memory in the auditory cortex, at least during the early stages of memory consolidation.
\end{abstract}

\section{Introduction}

Specific changes in the frequency receptive fields of individual auditory cortical neurons occur as a result of classical or instrumental conditioning (Diamond and Weinberger, 1986; Bakin and Weinberger, 1990; Edeline et al., 1993; Recanzone et al., 1993; Cahill et al., 1996; Weinberger, 2007). Furthermore, several studies suggest that experience with one sensory modality can influence the activity of cortical areas associated with other sensory modalities. For instance, in humans, auditory stimuli evoke responses in the visual cortex (McIntosh and Gonzalez-Lima, 1998; McIntosh et al., 1998; Zangenehpour and Zatorre, 2010), visual stimuli evoke or modulate responses in the auditory cortex (Bizley et al., 2007; Kayser et al., 2008), and responses of the auditory cortex to visual stimuli (and, conversely, responses of the visual cortex to auditory stimuli) increase after brief exposure to bimodal audiovisual stimuli (Meyer et al., 2007; Zangenehpour and

\footnotetext{
Received Aug. 24, 2012; revised March 25, 2013; accepted May 6, 2013.

Author contributions: X.C., Y.G., and J.H. designed research; X.C., Y.G., Z.L., J.F., Xinjian Li, and H.W. performed research; X.C., Y.G., Xiao Li, and J.H. analyzed data; X.C. and J.H. wrote the paper.

This work was supported by Hong Kong Research Grants Council (PolyU9/CRF/09) and Ministry of Science and Technology of China (2012CB966300). We thank Ray Guillery, Mu-ming Poo, and Bin Hu for their critical comments on this manuscript, Raymond Chung for advice on statistical analyses, and Eduardo Lau for administrative and technical assistance.

Correspondence should be addressed to Jufang He, Department of Rehabilitation Sciences, The Hong Kong Polytechnic University, Hung Hom, Kowloon, Hong Kong. E-mail: rsjufang@polyu.edu.hk.

Y. Guo's and H. Wang's present address: Institute of Biomedicine and Health, Chinese Academy of Sciences, Guangzhou, China.

Xinjian Li's present address: Krieger Mind/Brain Institute, Johns Hopkins University, Baltimore, MD 21218.

DOI:10.1523/JNEUROSCI.4078-12.2013

Copyright $\odot 2013$ the authors $\quad 0270-6474 / 13 / 339963-12 \$ 15.00 / 0$
}

Zatorre, 2010). Likewise, in monkeys, somatosensory (Zhou and Fuster, 2000) and prefrontal cortical neurons (Watanabe, 1992; Fuster et al., 2000) respond to both visual and auditory stimuli, auditory cortical neurons respond to visual stimuli after training in cross-modal associative tasks (Brosch et al., 2005; Brosch et al., 2011; Scheich et al., 2011), and exposure to an eight-degree mismatch between auditory and visual spatial inputs shifts the perception of auditory space (Recanzone, 1998). The association between different modalities has been found to occur at the single cell level in vitro and within the auditory cortex after repeated stimulation in vivo (Buonomano, 2003; Sugihara et al., 2006; Johnson et al., 2010). Taken together, these studies suggest that a cross-modal associative memory could be established using an appropriate training protocol.

Patients with damage to the medial temporal lobe show difficulty in forming new memories (Scoville and Milner, 1957; Corkin, 1984) but are able to recall remote memories (Teng and Squire, 1999; Wang et al., 2009; Lesburguères et al., 2011). By contrast, neocortical damage impairs remote memory (Graham and Hodges, 1997; Squire et al., 2001). According to the hippocampus-neocortex-transfer theory, memory is initially stored in the hippocampus and then gradually transferred to the neocortex, ultimately becoming independent of the hippocampus (Alvarez and Squire, 1994; Wiltgen et al., 2004). However, it is not yet known how the medial temporal lobe participates in the encoding or retrieval of new associative memories in the neocortex in an animal model.

Here, we established a cross-modal visuoauditory associative memory in the auditory cortex and determined that the resulting 
memory trace correlated with performance in behavioral tasks. First, using electrophysiological recordings in behaving rats, we monitored the encoding and extinction of visuoauditory memory traces in the auditory cortex. Next, we examined the effect of inactivating the entorhinal cortex on visuoauditory memory encoding and retrieval.

\section{Materials and Methods}

Animals. Subjects were male and female Sprague Dawley rats (280-360 g) with clean external ears and no observable behavioral abnormalities $(n=17)$.

Surgery. Rats were anesthetized with sodium pentobarbital $(50 \mathrm{mg} / \mathrm{kg}$, i.p.; Ceva Sante Animale). Anesthesia was maintained throughout surgery and pre-implantation recordings with $25 \mathrm{mg} / \mathrm{kg} / \mathrm{h}$ sodium pentobarbital. Atropine sulfate $(0.05 \mathrm{mg} / \mathrm{kg}$, s.c. $)$ was administered $15 \mathrm{~min}$ before the induction of anesthesia to inhibit tracheal secretions. Rats were mounted in a stereotaxic device, and a midline incision was made in the scalp after liberal application of a local anesthetic (xylocaine, 2\%). A craniotomy was performed at the temporal lobe approximately 3.0 to 5.0 $\mathrm{mm}$ from the top and bregma -3.0 to $-6.5 \mathrm{~mm}$ to access the auditory cortex, and the dura mater was removed. Body temperature was maintained at $37-38^{\circ} \mathrm{C}$ with a heating blanket. All procedures were approved by the Animal Subjects Ethics Sub-Committees of The Hong Kong Polytechnic University.

Before electrode implantation, tungsten microelectrodes with impedances of 2-7 M $\Omega$ (Frederick Haer) were used to map the frequencytuning properties of the auditory cortex. Electrodes were positioned with a stepping-motor microdriver controlled from outside the soundproofed room. Neuronal signals recorded by the microelectrode, together with auditory and visual signals, were amplified and stored using TuckerDavis Technologies (OpenEX, TDT) and Axoscope software (Molecular Devices).

We then implanted a homemade electrode array, typically consisting of four bundles of electrodes, into the auditory cortex of each hemisphere. One of the electrode bundles consisted of one recording electrode glued next to two stimulating electrodes. The other three bundles consisted of single recording electrodes. The stimulating electrodes were made of insulated stainless steel wire (A-M Systems) with an impedance of $<100 \mathrm{k} \Omega$. The recording electrodes were made of insulated tungsten wire (A-M Systems) with an impedance of 0.5-1 $\mathrm{M} \Omega$. One single-unit was isolated from each recording electrode at the stimulation site or from each recording electrode at the control site. The array was held by a micromanipulator and lowered to the surface of the cortex. After the electrode array arrived at the correct position, the skull opening was covered with a layer of silicone (World Precision Instruments). All electrodes were connected to a 20-pin socket that was cemented to the skull. After surgery, rats recovered in their home cages.

Auditory and visual stimuli. Auditory stimuli were digitally generated using a computer-controlled TDT Auditory Workstation and delivered through a coupled electrostatic speaker (EC1, TDT). The sound pressure level was calibrated with a condenser microphone (Center Technology). Pure tones of $60-70 \mathrm{~dB}$ SPL were used to map the tuning properties of the auditory cortex before electrode implantation in anesthetized rats, whereas white noise (60-70 dB SPL) was used for the conditioning experiments with behaving rats. White light generated by light-emitting diodes placed $40 \mathrm{~cm}$ above the experimental chamber was used as the visual stimulus. When the light was on, the illumination at the bottom of the chamber was 26 Lux.

Association of auditory and visual stimuli. Rats were presented with a combined stimulus consisting of a $100 \mathrm{~ms}$ light followed by electrical stimulation of the auditory cortex (two pulses at $30-100 \mu \mathrm{A}$ ) at a $200 \mathrm{~ms}$ delay. We chose to deliver the visual stimulus $200 \mathrm{~ms}$ before electrical stimulation of the auditory cortex for two reasons: (1) to allow time for the visual signal to reach the auditory cortex, which was the focus of our electrophysiological experiments; (2) to increase the likelihood that rats could detect a time difference in the onset of the two stimuli, which may increase their attention to the stimuli during serial presentation. The combined stimulus was either presented alone $(n=4)$ or was followed by a foot shock ( $600 \mathrm{~ms} ; 0.5-0.9 \mathrm{~mA})$ at a $300 \mathrm{~ms}$ delay $(n=8)$. Either 15 or 20 stimulus presentations occurred in each session. The pulses used to activate the auditory cortex were generated by the TDT system and delivered through the stimulation electrode (of low impedance) via an isolator (ISO-Flex, A.M.P.) (He, 1997; Y. Q. Yu et al., 2004; X. J. Yu et al., 2009). Several conditioning sessions were conducted each day with intervening test sessions. The total numbers of trials for each experiment are shown in the figures. To examine the encoding and extinction of the visuoauditory association, test trials were inserted between training sessions and also administered at different delays after training. Test trials were typically comprised of 10 presentations of the visual stimulus. We recorded neuronal responses to the visual stimulus and the amount of time rats spent freezing during the $30 \mathrm{~s}$ after the onset of each visual stimulus.

Reward retrieval. In some experiments, $2 \mathrm{~d}$ after visuoauditory conditioning, rats underwent reward retrieval training, during which they learned to retrieve a water reward after sensing electrical stimulation of the auditory cortex. After water intake was restricted $(70 \%$ of normal intake), rats were placed in a homemade cage with three horizontally aligned holes (Otazu et al., 2009). An infrared sensor in the center hole detected nose-poking. Rats nose-poked in the center hole to initiate a trial, after which they were presented with electrical stimulation of the auditory cortex. Another infrared sensor in the right hole detected whether the rat arrived within $2 \mathrm{~s}$ of the onset of the electrical stimulation, in which case a water reward delivered. The left hole was not used.

Training occurred across five stages. In stage 1, rats were trained to obtain water from the right hole. In stage 2 , rats were required to nosepoke in the center hole to receive a water reward in the right hole. In stages $3-5$, rats were trained to keep their noses in the center hole for varying amounts of time before stimulus presentation to receive the reward. The holding time varied randomly from 200 to $800 \mathrm{~ms}$ (stage 3), 400 to $1500 \mathrm{~ms}$ (stage 4), and 100 to $1200 \mathrm{~ms}$ (stage 5). Rats progressed to the next stage after they successfully obtained the reward in four successive trials. In stage 5 , the performance criterion was the successful retrieval of the reward in nine successive trials. Because rats nose-poked in the center hole to initiate a trial and retrieved the reward after sensing electrical stimulation, we named this task the subject-initiated cortical stimulation-reward protocol.

One day after reaching criterion in the subject-initiated cortical stimulation-reward protocol, rats were trained in the same general protocol except that the electrical cortical stimulation was replaced with the visual light stimulus. We reasoned that the previously-established association between the light and cortical stimulation would lead rats to continue to perform the task successfully. This task was named the subject-initiated light-reward (SiLR) protocol. We categorized trials as successful if rats triggered the light and moved to the right hole for the reward, unsuccessful if rats triggered the light but failed to move to the right hole for the reward, or premature if rats nose-poked in the center hole but failed to trigger the light. We computed success rate as the number of successful trials divided by the sum of successful and unsuccessful trials. We also examined whether rats would approach the right hole for the reward if they were exposed passively to the visual light stimulus, which was called the experimenter-initiated light-reward (EiLR) protocol.

Electrophysiological examination of the visuoauditory association. After rats recovered from surgery $(6-7 \mathrm{~d})$, we recorded neuronal responses to the auditory, visual, and electrical stimuli at the stimulation and control sites via the implanted electrode array. Neurons that were located close to the stimulating electrode and showed responses to the electrical stimulation were considered as being in the stimulation site, whereas those that were located $>1 \mathrm{~mm}$ from the stimulating electrode and showed no response to stimulation were considered as being in the control site. In the first electrophysiological experiment $(n=9)$, we examined simple visuoauditory association by recording neuronal responses to the light after conditioning. In the second electrophysiological experiment $(n=$ 8 ), we examined the time course of the establishment of the artificial visuoauditory association by randomly inserting 10 test trials (i.e., light stimulus only) into the light-cortical stimulation-foot shock protocol. In the third electrophysiological experiment $(n=4)$, we recorded neuronal responses to the light while rats were performing the SiLR or EiLR pro- 
A Schematic drawing of electrode implantation

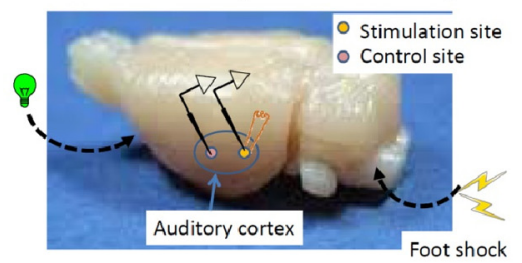

2 Sequence of stimuli in conditioning with foot shock

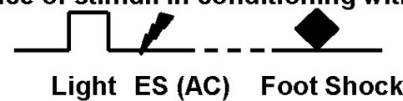

B

1 Stimulation site

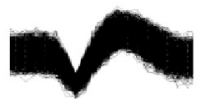

2
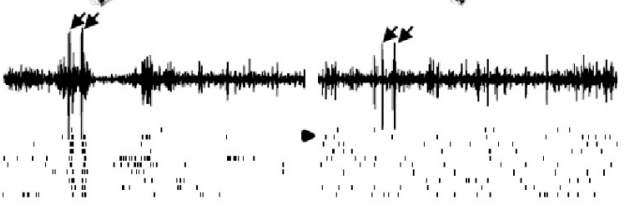

3
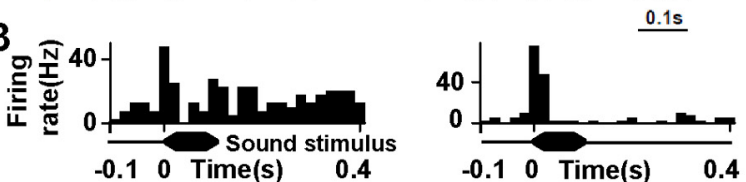

$\mathbf{C}_{1}$

Before conditioning

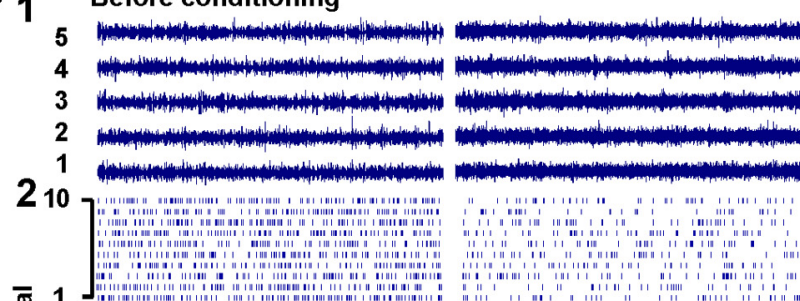

A 1 After conditioning

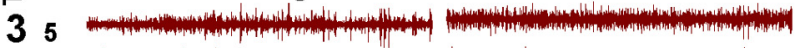

4 .

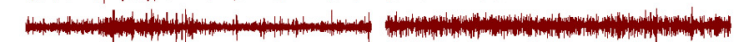

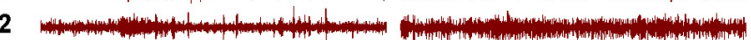

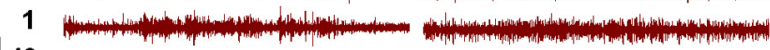

410
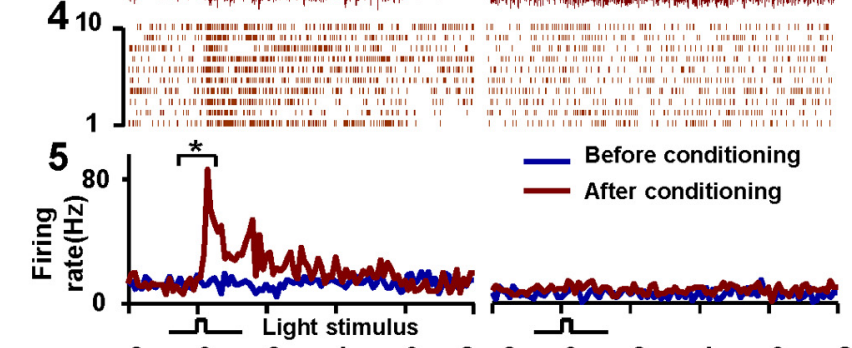

D

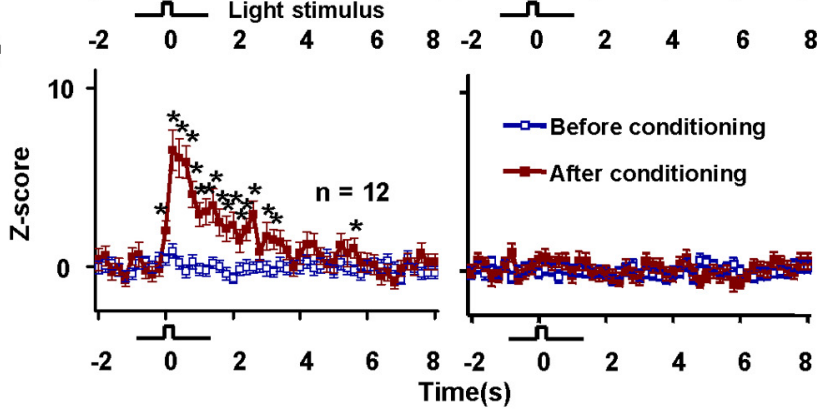

E

1 Stimulation site Control site

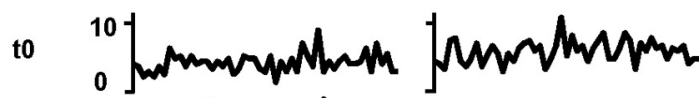

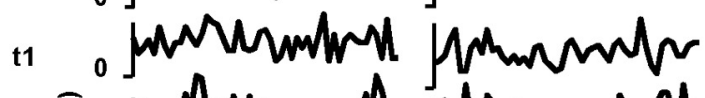

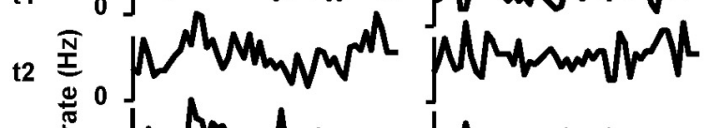

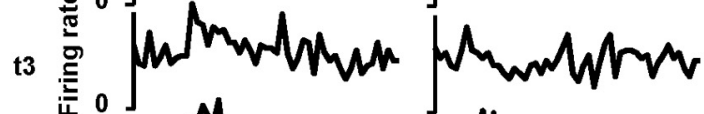

t4

t5
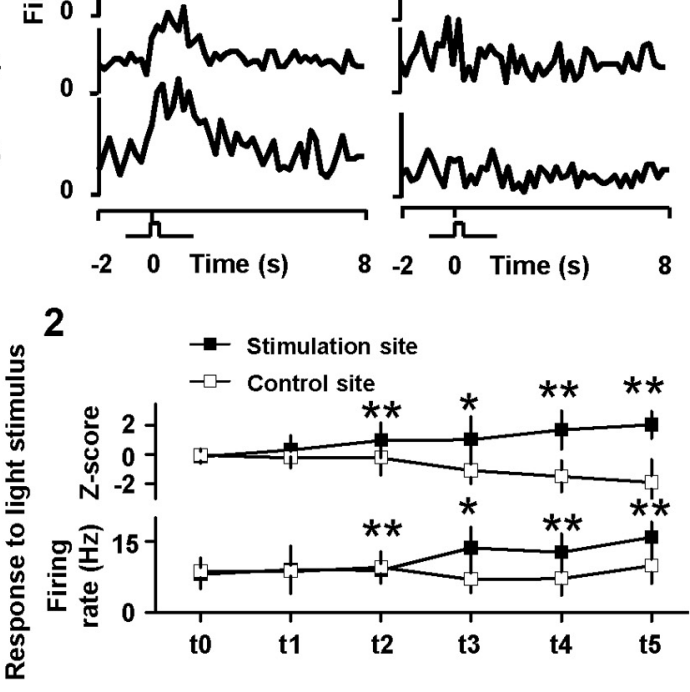

3

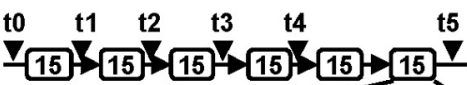

$\nabla$ Test (15 trials)

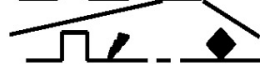

15 Conditioning (15 trials) Light ES Foot Shock

$\mathbf{F}$

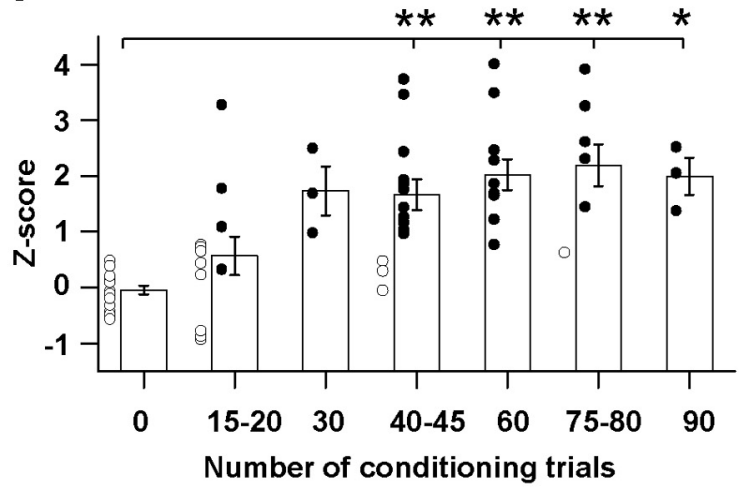

Figure 1. Auditory cortex neuronal responses to a visual stimulus after pairings of a compound visual stimulus/electrical cortical stimulation with foot shock. A1, Electrode bundles, consisting of a pair of stimulating and recording electrodes at the stimulation site and a recording electrode at the control site, were implanted in the auditory cortex. A2, Sequence of stimulus presentations during conditioning. B1, Spike profile of single-unit neurons at stimulation and control sites. B2, Neuronal responses to electrical cortical stimulation. The left signal was (Figure legend continues.) 
tocol. In the fourth electrophysiological experiment $(n=4)$, we examined extinction of an established association by repeating the SiLR and EiLR protocol across days.

Inactivation of entorhinal cortex. To examine the role of the medial temporal lobe on the encoding and retrieval of associative memory in the auditory cortex, we implanted a guide cannula $(0.48 \mathrm{~mm}$ in diameter; RWD Life Science) unilaterally into the entorhinal cortex (from bregma: AP $-6.6 \mathrm{~mm}$ and ML $4.7 \mathrm{~mm}$; depth from skull surface: $6.8 \mathrm{~mm}$ ). The drug infusion site was determined from a pilot study conducted in anesthetized rats, in which activation of this region modulated auditory cortex neuronal responses to an auditory stimulus. The guide cannula was cemented to the skull with the stimulating and recording electrode matrices. Patency of the cannula was maintained by inserting a dummy needle extending $0.2 \mathrm{~mm}$ below the guide.

For drug infusion, we connected an injection needle $(0.3 \mathrm{~mm}$ in diameter; RWD Life Science) extending $0.7 \mathrm{~mm}$ below the tip of the guide to a Hamilton syringe. To examine the role of the entorhinal cortex in memory encoding, we inactivated the entorhinal cortex by infusing either lidocaine $(2 \%, 2 \mu \mathrm{l}, 0.5 \mu \mathrm{l} / \mathrm{min} ; n=1)$ or DNQX, an AMPA receptor antagonist (15 $\mathrm{mM}, 1.2 \mu \mathrm{l}, 0.3 \mu \mathrm{l} / \mathrm{min} ; n=3), 15 \mathrm{~min}$ before three 60 -trial conditioning sessions (i.e., pairing light/cortical stimulation with foot shock). On the following day, another 60-trial session was administered to all rats with no drug infusion. An additional session of 40 trials was further administered to one rat with no drug infusion on another day. To examine the role of the entorhinal cortex in memory retrieval, we infused DNQX $(n=5) 15$ min before testing. Microinjections were controlled by a Quintessential Stereotaxic Injector (QSI, Stoelting). The injection needle was left in place for $5 \mathrm{~min}$ after the completion of infusion. One to three monitoring electrodes were implanted $\sim 1.0$ to $2.0 \mathrm{~mm}$ away from the drug infusion cannula to confirm the effectiveness of the drug infusion on entorhinal cortex activity. As the objective of this experiment was to reversibly inactivate the entorhinal cortex (i.e., to compare memory encoding and retrieval with and without drug infusion within the same rats) and not to compare the effects of different drugs, no vehicle control group was used.

After visuoauditory associative memory was established in the auditory cortex (i.e., when neurons started showing responses to the visual

\section{$\leftarrow$}

(Figure legend continued.) recorded by an electrode at the stimulation site, and the right signal was recorded by an electrode at the control site. Arrows indicate artifacts of the stimulation pulses, which were of moderate amplitude and easily separated from neuronal signals. Raster plots show neuronal responding over 10 trials. Arrowheads indicate the trials shown in the raw data samples. Artifacts are not included in the raster displays. B3, PSTHs show auditory cortex neuronal responses to a sound stimulus. $C$, Neuronal responses at the stimulation and control sites to a repeated visual stimulus before conditioning [blue: (1) raw data, (2) raster plots, (5) PSTHs] and after conditioning [red: (3) raw data, (4) raster plots, (5) PSTHs]. Raw data are shown only for five stimulus presentations before and after conditioning. Neuronal activity during a 2 s period immediately preceding the onset of the visual stimulus was compared with that during a $2 s$ period immediately after the visual stimulus $\left({ }^{*} p<0.001, t\right.$ test). $D, Z$-scores (mean $\pm \mathrm{SE}$ ) of neuronal responses to the visual stimulus before and after conditioning (12 pairs of neurons at stimulation and control sites sampled from nine rats). Each data point represents $200 \mathrm{~ms}$ of recording time. Neuronal responses before conditioning were compared with those after conditioning for both stimulation and control sites $\left({ }^{*} p<0.05\right.$, one-way ANOVA). $\boldsymbol{E}$, Time course of the establishment of an associative memory trace in the auditory cortex. The representative pair of neurons was chosen from those whose activity is shown in $\boldsymbol{D}$ but was not the same as those whose activity is shown in $\boldsymbol{B}$ and $\boldsymbol{C}$. $\boldsymbol{E 1}$, Neuronal responses to the visual stimulus before ( $\mathrm{t} 0$ ) and after each conditioning block ( $\mathrm{t} 1-\mathrm{t} 5)$; each block consisted of 15 pairings of the visual stimulus/cortical stimulation with foot shock. Histograms are based on data from 15 repeated testing trials. E2, Changes in neuronal responding (Z-scores and firing rates) during the $2 \mathrm{~s}$ period after the onset of the visual stimulus $\left({ }^{*} p<0.05,{ }^{* *} p<0.01\right.$, paired $t$ test; neuronal responses to the visual stimulus vs spontaneous firing). $\mathbf{E 3}$, Experimental timeline. $\boldsymbol{F}$, The establishment of an associative memory trace in the auditory cortex across conditioning trials with a foot shock. Scatter shows Z-scores of individual neuronal responses to the test visual stimulus ( $n=16$ neurons from eight rats). Neurons showing significant responses to the visual stimulus were plotted with filled circles ( $p<0.05, t$ test), and those showing no significant responses were plotted with open circles. Bar graph shows mean Z-scores ( $\pm \mathrm{SE}$ ) of neuronal responses, which were compared with those before conditioning $(F=9.715, p<$ 0.0001 , one-way repeated-measures ANOVA; ${ }^{*} p<0.05$, ${ }^{* *} p<0.01$, post hoc). stimulus), we examined neuronal responses when the entorhinal cortex was either inactivated or intact across subsequent days in a pseudorandom order. The experiment continued for 4 to $12 \mathrm{~d}$ until no signal was detectable or the fear memory was extinguished.

All of the recordings in the present study were carried out in freely moving rats unless otherwise specified. The percentage of time rats spent freezing was calculated during the $30 \mathrm{~s}$ after the onset of each visual stimuli and used as a behavioral index of fear associated with the visual stimulus.

Data analysis. Auditory cortex neuronal responses and behavioral responses were recorded simultaneously using a computer. Single-unit spikes were distinguished using spike sorting software (OpenSorter, TDT and SPKtool, a MATLAB open source software, MathWorks). We considered 3 SDs above baseline as the threshold to distinguish spikes. A template matching method in SPKtool or a K-means clustering method in OpenSorter was adopted to sort single-unit spikes. The unit with the largest amplitude and normal overlaid spike profile was chosen from each electrode. Another criterion was that the number of spikes with an interspike interval of $<2 \mathrm{~ms}$ in the histogram should be $<0.2 \%$ of the total number of spikes. The timing of spike occurrence relative to stimulus delivery was calculated using MATLAB software. Peristimulus histograms (PSTHs) were calculated over a bin size of $20 \mathrm{~ms}$ for auditory responses and 100 or $200 \mathrm{~ms}$ for visual responses.

Because there was no guarantee of recording from the same unit across days, we used $Z$-scores (mean \pm SE) to examine neuronal responses and compare responses across sessions (Otazu et al., 2009). Z-scores of neuronal responses to visual stimuli within a certain time period were calculated against the mean spontaneous firing rate within the same period, thereby representing the distance between the neuronal responses and the mean of spontaneous firing in units of $\mathrm{SD}(\mathrm{Z}=(x-\mu) / \delta$; where $x$ was the neuronal response in each trial, and $\mu$ and $\delta$ were the mean and $\mathrm{SD}$, respectively, of the spontaneous firing rates across all trials). Higher $Z$-scores typically indicate a larger neuronal response to the stimulus, although they also differed depending on the total number of testing trials.

Changes in $Z$-scores after each conditioning session were used to examine the effectiveness of conditioning to induce neuronal plasticity. Paired Student's $t$ tests were used to compare neuronal responses with spontaneous neuronal activity. One-way repeated-measures ANOVA was used to test for differences in mean $Z$-scores before and at different times after conditioning sessions. One-way ANOVA was used to test for differences in mean $Z$-scores among different treatment groups. Statistical significance was set at $p<0.05$.

\section{Results}

\section{Artificial association of visual and auditory stimuli}

We established an artificial association between a visual stimulus (i.e., light) and electrical stimulation of the auditory cortex by pairing a compound visual stimulus/cortical stimulation with foot shock in freely moving rats (Fig. 1 A1-2). Electrical stimulation of the auditory cortex by a stimulating electrode evoked neuronal responses that were detected by a closely attached recording electrode (Fig. 1B2, left). Another recording electrode placed 2-3 mm away from the stimulating electrode served as a control site (Fig. 1 B2, right), at which neurons were expected to respond to sound (Fig. 1 B3, right) but not electrical stimulation (Fig. 1 B2, right raster plot). All data were sorted to single-units (Fig. 1B1). We used electrical stimulation instead of a sound stimulus because electrical stimulation directly activates a smaller cortical area than does sound, allowing us to monitor the activated area via a recording electrode in close proximity to the stimulating electrode.

Before conditioning, neurons at both the stimulation and control sites responded to a sound stimulus (white noise; Fig. $1 B 3$ ) but not the visual stimulus (Fig. 1C1-2). After eight blocks of 15 conditioning trials, neurons at the stimulation site but not the control site responded to the visual stimulus (Fig. 1C3-4), 

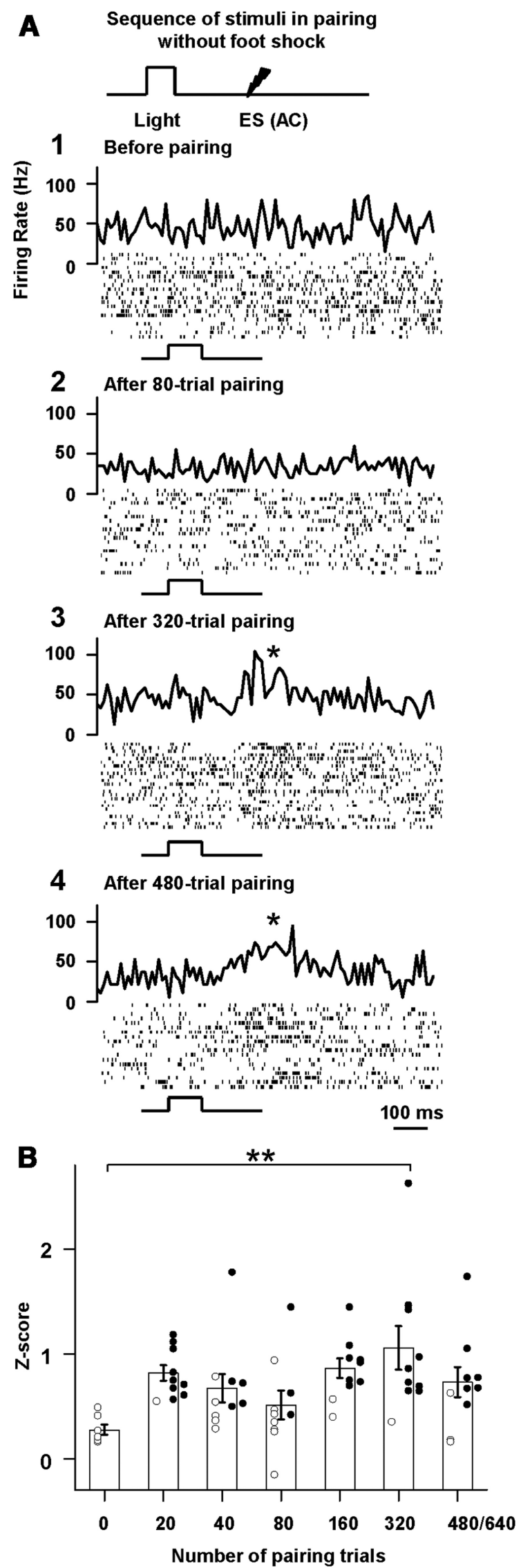

Figure 2. Auditory cortex neuronal responses to a visual stimulus after pairing the visual stimulus with electrical stimulation of the auditory cortex without foot shock. $A$, Neuronal responses (PSTHs and raster plots) to repeated visual stimuli before and after pairing of the providing evidence of a visuoauditory association that was observable in the raw recording signal (Fig. 1C3). Across 10 testing trials, postconditioning neuronal responses at the stimulation site during the $2 \mathrm{~s}$ period immediately after the onset of the visual stimulus were significantly greater than those during the $2 \mathrm{~s}$ baseline period immediately before the visual stimulus (one neuron was selected at each site using spike sorting; spikes are shown in the raster plots, Fig. $1 C 2,4 ; p<0.001, t$ test, Fig. 1C5). Responses to the visual stimulus at the stimulation site increased significantly after conditioning ( $Z$-scores, $9.60 \pm 0.99$ vs $0.13 \pm 0.56$, $p<0.001, t$ test), whereas responses to the visual stimulus at the control site were unchanged $(0.67 \pm 0.65$ vs $0.25 \pm 0.48, p=$ $0.66, t$ test). After conditioning, mean $Z$-scores of neuronal responses after the onset of the visual stimulus were significantly greater than those during the baseline period, but only at the stimulation site and not at the control site (Fig. $1 D, n=12$ pairs of neurons from eight rats; $F=45.19, p<0.00001$, one-way repeated-measures ANOVA; ${ }^{\star} p<0.05$, post hoc; one or two pairs of neurons sampled from each rat).

Time course of the encoding of the artificial visuoauditory association

Next, we examined the time course of visuoauditory memory encoding by inserting visual stimulus test trials between the conditioning sessions (Fig. 1E3). Recordings were taken from two electrodes, one at the stimulation site and one at the control site. Before conditioning, neurons at both electrodes showed no response to the visual stimulus (Fig. $1 \mathrm{E} 1 ; \mathrm{t} 0$ ). During conditioning, neurons at the stimulation site did not respond to the visual stimulus after the first block ( 15 trials; $\mathrm{t} 1$ ) but started to respond after the second block ( 30 trials; 2 ) and continued to respond across all subsequent blocks ( $\mathrm{t} 3-\mathrm{t} 5$; Fig. 1 E1). Beginning at $\mathrm{t} 2$ and continuing through $\mathrm{t} 5$, both $Z$-scores and firing rate to the visual stimulus were significantly greater than those associated with spontaneous firing (Fig. $1 E 2,{ }^{\star} p<0.05,{ }^{*} p<0.01, t$ test). By contrast, neurons at the control site did not respond to the visual stimulus at any time point (Fig. 1E1-2, right).

To determine the time course of artificial memory encoding, rats were given 15 or 20 trials per conditioning session. All data were obtained within $1 \mathrm{~d}$, except for one rat that was trained for $2 \mathrm{~d}$. Mean $Z$-scores of visual stimulus-evoked responses were significantly greater than baseline responses after 40 or 45 trials (Fig. $1 F,-0.047 \pm 0.077$ vs $1.67 \pm 0.28$; one-way ANOVA, post hoc, $p<0.001, n=16)$ and became stable there after $(F=9.715$, $p<0.0001$; one-way ANOVA). Four neurons started to respond to the visual stimulus after 20 trials, and all neurons showed visual stimulus-evoked responses after 30 trials.

Visuoauditory association without foot shock

To examine whether foot shock was necessary to establish the visuoauditory association, we presented the combined stimulus

\footnotetext{
$\leftarrow$

visual stimulus with electrical stimulation of the auditory cortex. Neuronal activity during a 150 ms period after the onset of the visual stimulus was compared with that during a $150 \mathrm{~ms}$ period immediately preceding the visual stimulus $\left({ }^{*} p<0.05, t\right.$ test). $\boldsymbol{B}$, Time course of the establishment of an associative memory trace in the auditory cortex. Scatter shows Z-scores of individual neuronal responses to the test visual stimulus ( $n=10$ neurons from four rats) after different numbers of conditioning trials without foot shock. Neurons showing significant responses to the visual stimulus were plotted with filled circles ( $p<0.05, t$ test), and those showing no significant responses were plotted with open circles. Bar graph shows the mean Z-scores ( \pm SE) of neuronal responses after conditioning, which were compared with those before conditioning $\left(F=3.112, p<0.01\right.$, one-way ANOVA; ${ }^{* *} p<0.01$, post hoc $)$.
} 
A

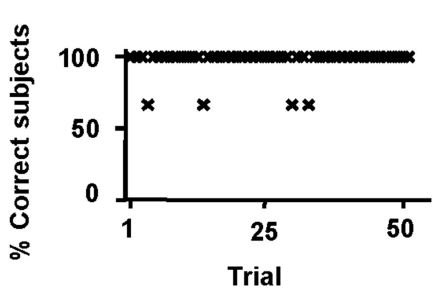

C

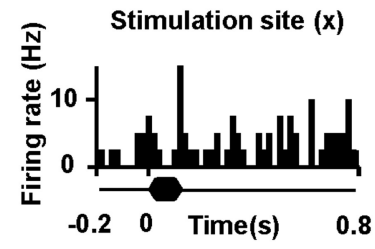

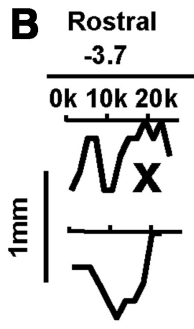

$-5.1$

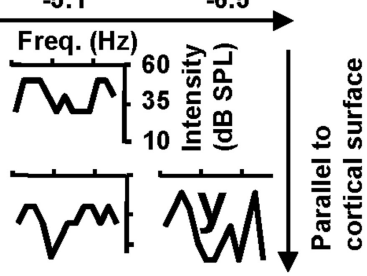

Control site (y)

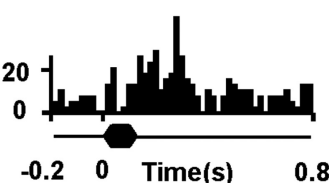

D Before conditioning (BC)

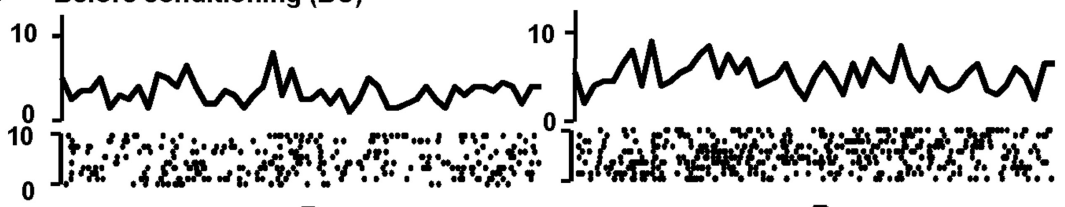

$\Omega$

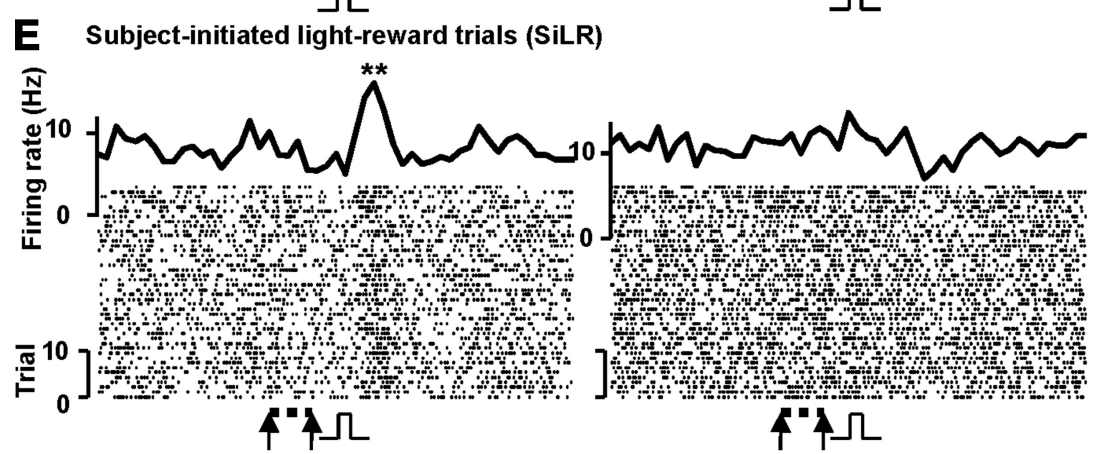

F Experimenter-initiated light-reward trials (EiLR)

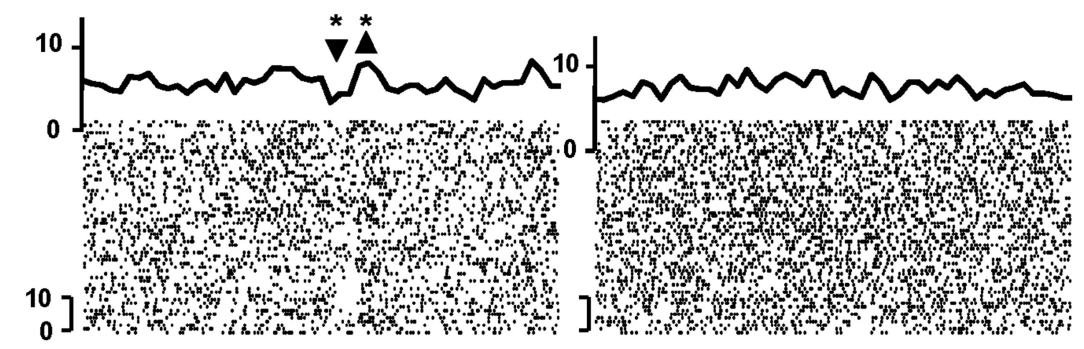

几

几

G Subject-initiated premature trials (SiP)

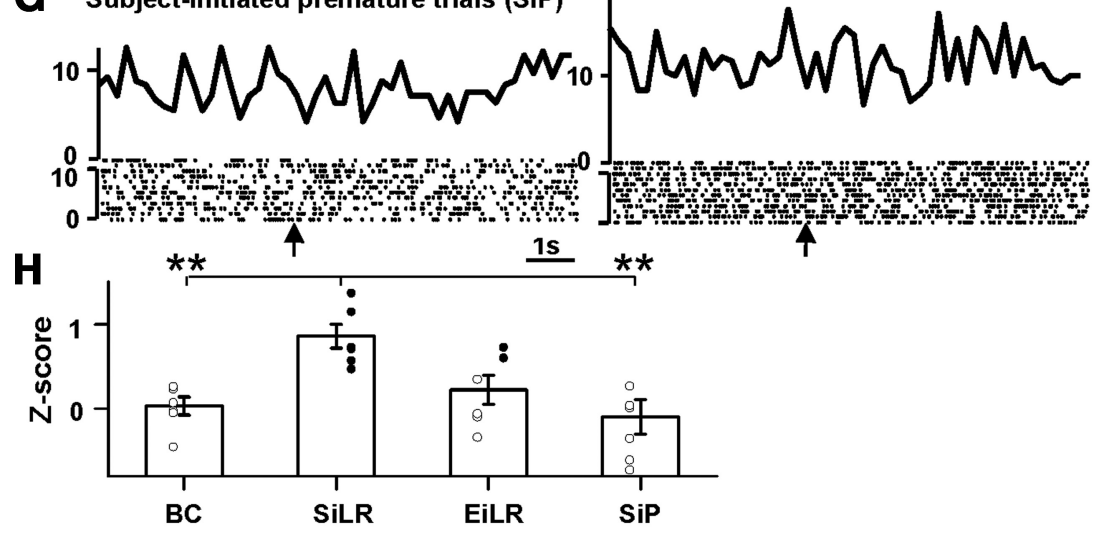

Figure 3. Electrophysiological measurement of the artificial visuoauditory association during reward retrieval. $\boldsymbol{A}$, Percentage of rats that successfully retrieved the reward after the visual stimulus in the SiLR protocol. $\boldsymbol{B}$, Tuning curves were obtained in anesthetized rats before electrode array implantation. Labels of intensity and frequency coordinates apply to all curves. Coordinates

(light and electrical stimulation of the auditory cortex) without foot shock to behaving rats. Neurons showed no response to the visual stimulus before pairing (Fig. $2 A 1$ ) or after 80 trials (Fig. 2A2), but did respond to the visual stimulus after 320 and 480 trials $(p<0.05, t$ test; Fig. 2A3-4)

Of the 10 single-units sampled from rats, 9 showed responses to the visual stimulus after 20 trials, whereas 5, 3, 8, 9, and 7 neurons showed responses after 40 , $80,160,320$, and 480/640 trials, respectively (Fig. $2 B$ ). $Z$-scores of responses to the visual stimulus after 320 trials were significantly greater than those before stimulus pairing $(F=3.122, p<0.01$, one-way ANOVA; $p<0.01$, post hoc). Therefore, foot shock was not necessary to induce the visuoauditory association, although memory encoding was faster and more predictable with foot shock.

Behavioral confirmation of the visuoauditory association

We next examined whether the visuoauditory associative memory could be behaviorally expressed in a non-fear-related task. After using classical fear conditioning to establish an association between the visual stimulus and electrical stimulation of the auditory cortex, rats were trained in an operant reward task to examine whether they could successfully recall and use the previously established visuoauditory association to retrieve a water reward. In this subjectinitiated cortical stimulation-reward proto-

\footnotetext{
and spacing of sampled neurons are shown with the locations of the stimulating $(x)$ and recording $(y)$ electrodes. C, Neuronal responses to the auditory stimulus at the stimulation and control sites before conditioning. $D$, Neuronal responses to the visual stimulus at the stimulation and control sites BC. $E$, Neuronal responses during the SiLR protocol. Only trials in which the rat successfully triggered the visual stimulus and retrieved the reward were included. Arrows show the times at which rats initiated a trial by nose-poking in the center hole. Square waves show the triggered visual stimulus. Neuronal responses during the $1 \mathrm{~s}$ after the visual stimulus were compared with spontaneous activity immediately before the visual stimulus $\left({ }^{* *} p<0.01\right.$, paired $t$ test). $\boldsymbol{F}$, Neuronal responses during the EiLR protocol. G, Neuronal activity during trials in which rats failed to trigger the visual stimulus (SiP). Arrows indicate when the rat nose-poked in the center hole. $\boldsymbol{H}, \mathbf{Z}$-scores of neuronal responses to the visual stimulus $B C$ and in different protocols (SiLR, EiLR, and SiP). Scatter shows Z-scores of individual neurons, and the bar graph shows mean $( \pm S E)$ $Z$-scores. Neurons showing significant responses to the visual stimulus were plotted with filled circles ( $p<0.05, t$ test) and those showing no significant responses were plotted with open circles. Mean Z-scores were significantly different among different protocols $(F=9.407, p<0.0001$, one-way ANOVA; ${ }^{* *} p<0.01$, post hoc)
} 

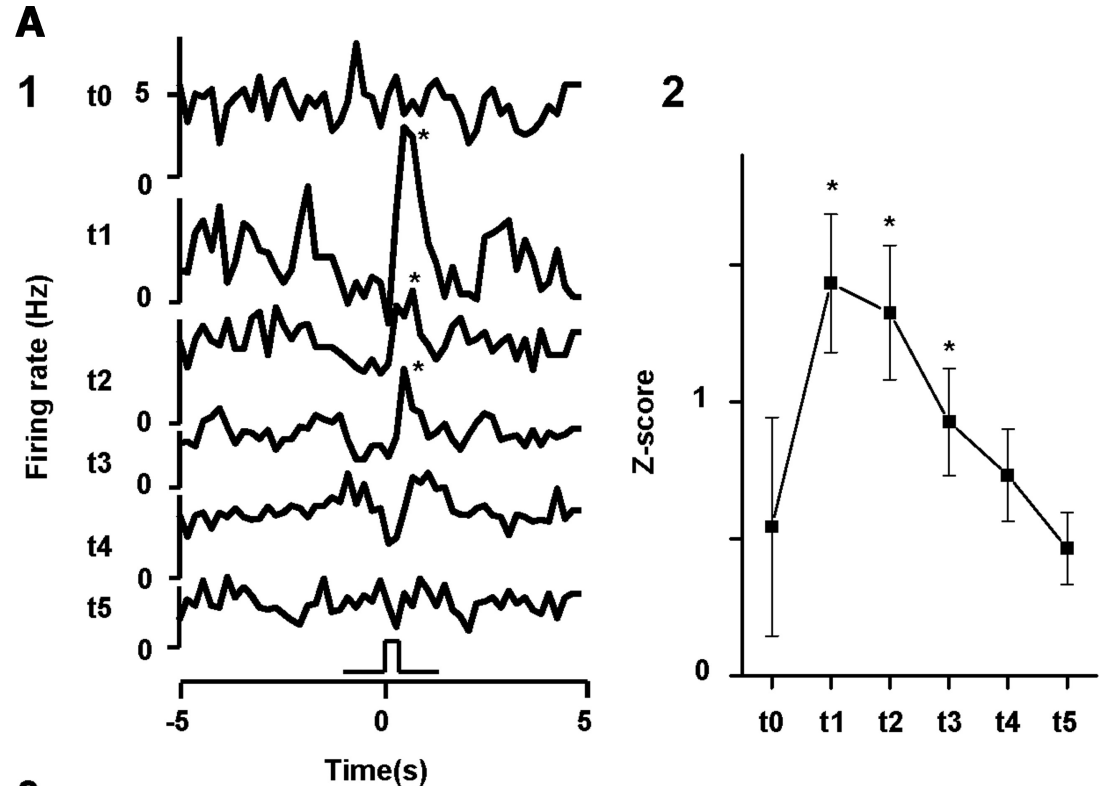

3

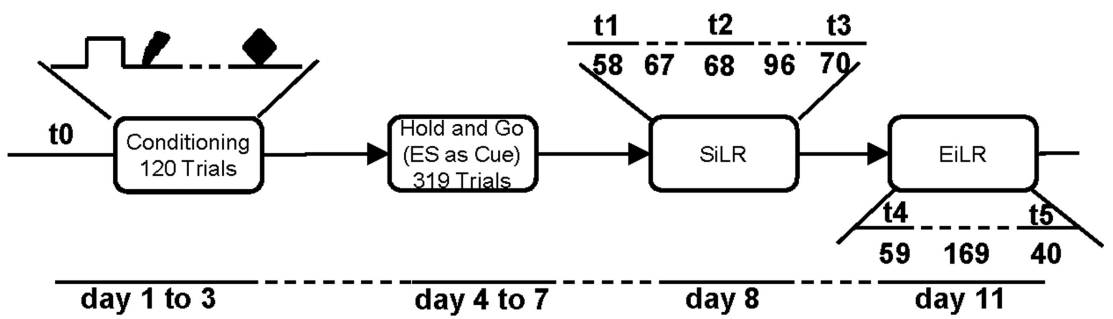

B

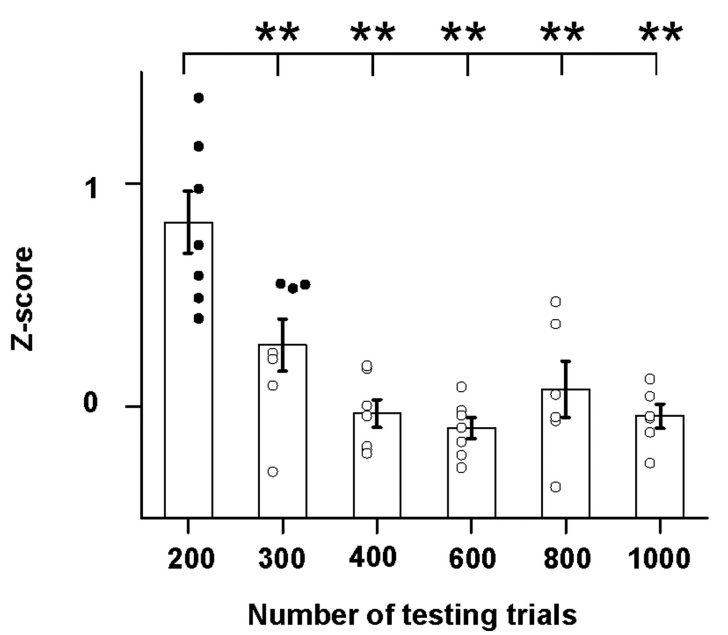

Figure 4. Extinction of the artificial visuoauditory association. A1, PSTHs of neuronal responses to the visual stimulus before conditioning (t0) and at different times after conditioning (t1-t5). $\mathbf{A 2}, Z$ - -scores of neuronal responses to the visual stimulus before and at different times after conditioning ( ${ }^{*} p<0.05$, paired $t$ test; neuronal responses to the visual stimulus vs spontaneous firing). $\boldsymbol{A}$ 3, Experimental timeline. $\boldsymbol{B}$, Neuronal responses to the visual stimulus after different numbers of test trials with the visual stimulus. Neurons showing significant responses to the visual stimulus were plotted with filled circles $(p<0.05, t$ test), and those showing no significant responses were plotted with open circles. Mean Z-scores were significantly different across different numbers of testing trials $\left(F=12.90, p<0.0001\right.$, one-way ANOVA; ${ }^{* *} p<0.01$, post hoc).

col, rats were required to nose-poke in a center hole for a varying duration of 100-1200 ms before electrical auditory cortex stimulation was triggered to ensure that cortical stimulation was used as a cue for reward availability. Across five stages of training, all rats learned to retrieve the reward after cortical stimulation. Subsequently, we replaced cortical stimulation with the visual stimulus. During the first trial with the visual stimulus, all four rats successfully retrieved the reward (Fig. 3A). When the visual stimulus was triggered by the experimenter (EiLR), all rats moved to the right hole for the reward, indicating that they used the previously established visuoauditory association to determine reward availability.

\section{Electrophysiological responses during reward retrieval}

In the same rats as above, which had implanted stimulating/recording electrodes at the stimulation site and recording electrodes at the control site (Fig. $3 B$ ), we examined auditory cortical neuronal responses during reward retrieval. Although no discrimination between primary and association auditory cortices was made for most rats, the stimulation site showed a multi-peaked frequency tuning curve (Fig. 3B) and thus was likely in the association auditory cortex. Before conditioning, neurons at both stimulation and control sites responded to sound (Fig. 3C) but not the visual stimulus (Fig. 3D).

These rats had been exposed to 120 pairings of a compound visual stimulus/ cortical stimulation with foot shock. During the SiLR protocol, neurons at the stimulation site responded to the visual stimulus when the rat initiated the trial, whereas neurons at the control site did not (Fig. 3E,H). Likewise, during the EiLR protocol, neurons at the stimulation site responded to the visual stimulus, whereas neurons at the control site did not (Fig. 3F,H). Responses consisted of two components: an early inhibitory component within $1 \mathrm{~s}$ and a later excitatory component (marked by two arrowheads in Fig. 3F). Auditory cortex activity was briefly inhibited when the visual stimulus was triggered by the experimenter, particularly during the first 20 trials (Fig. $3 F)$. This inhibition may have been caused by modulation of higher cortical centers when rats were passively presented with a cue associated with a reward.

Occasional trials in which rats failed to trigger the visual stimulus before attempting to retrieve the reward were called subject-initiated premature (SiP) trials. During these trials, neurons at both the stimulation and control sites showed no responses to the visual stimulus during the $2 \mathrm{~s}$ period after nose-poking (Fig. $3 G, H)$. Therefore, these trials served as a control indicating that the responses during SiLR and EiLR trials (Fig. $3 E, F$ ) were not induced by reward retrieval movements. All neurons responded to the visual stimulus during the SiLR protocol, whereas none responded to the visual stimulus before conditioning (BC) or during SiP trials (Fig. $3 H$ ). Mean $Z$-scores were significantly greater during SiLR trials than $\mathrm{BC}$ or during SiP trials $(n=6 ; F=$ 
Drug injection site in
entorhinal cortex (EC)

Subject \#2

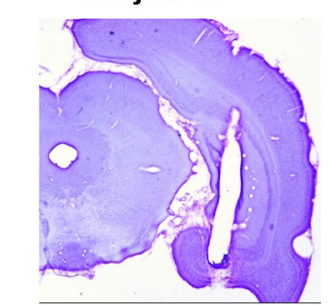

\#4

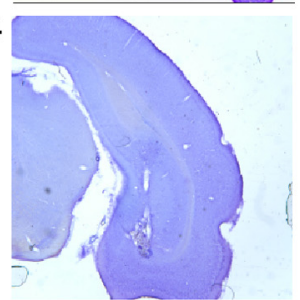

B

\#4 before drug Injection after drug Injection
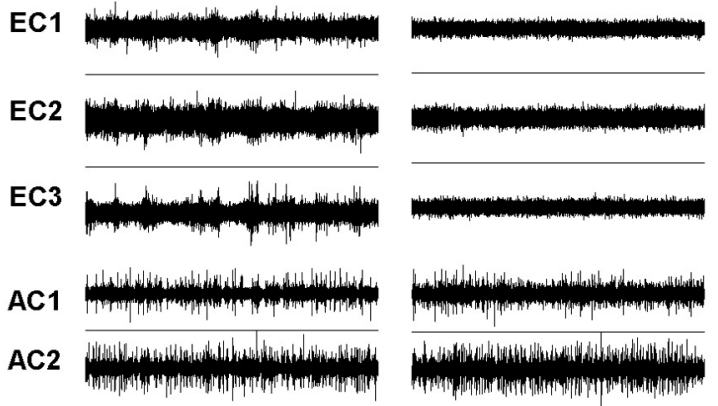

C

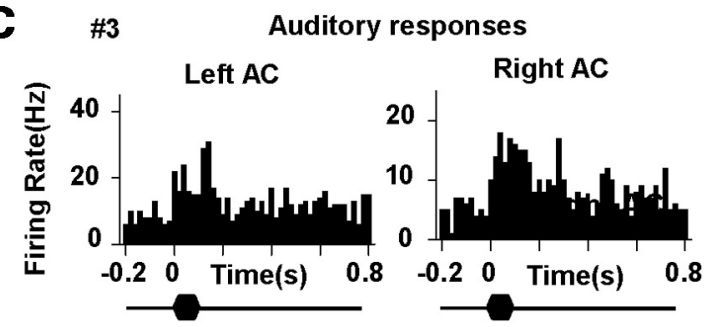

D

Responses to electrical stimulation of left AC

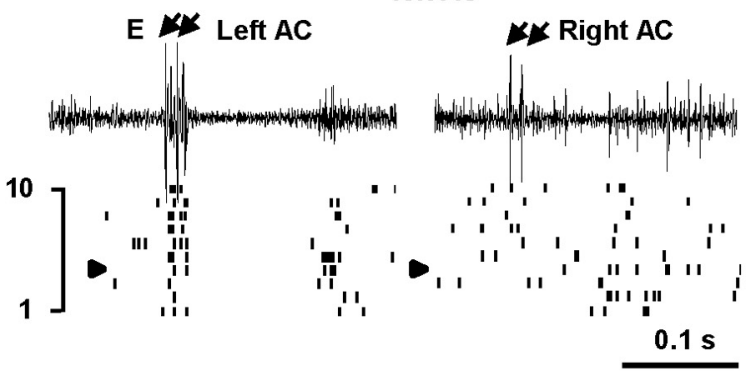

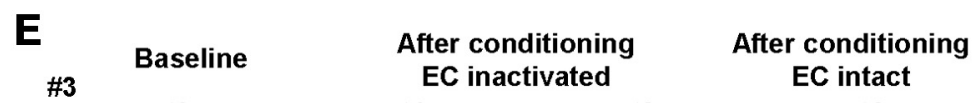

t1

t3

EC intact
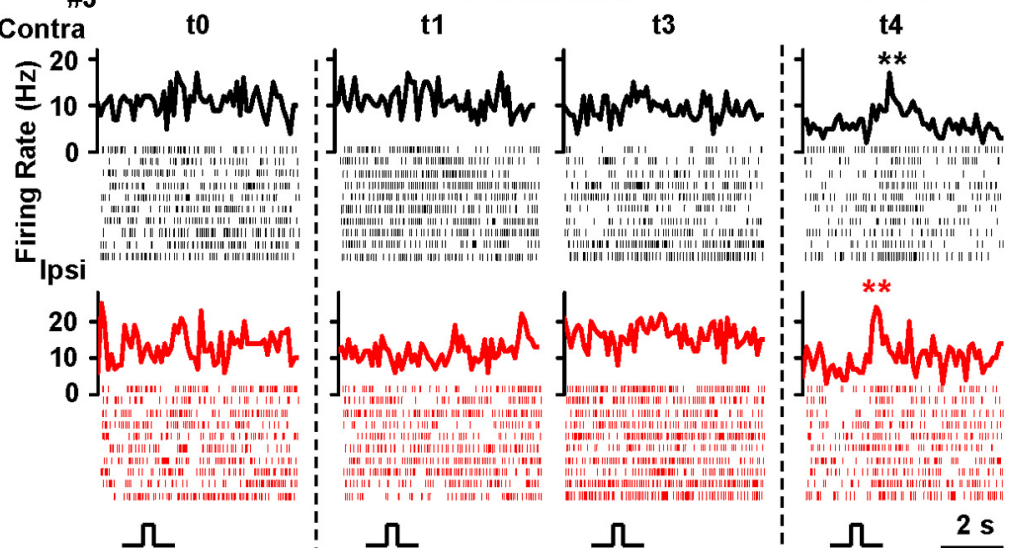

$\mathbf{F}$
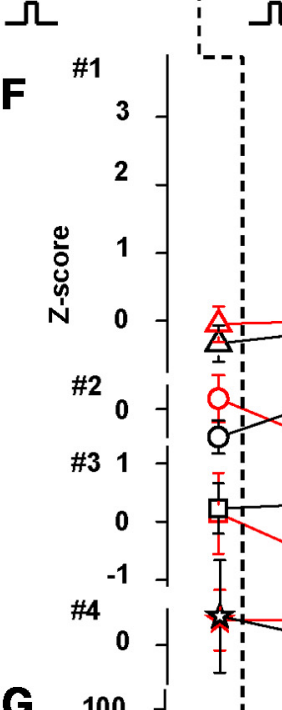

$\Omega$

$\Omega \quad 2 \mathrm{~s}$

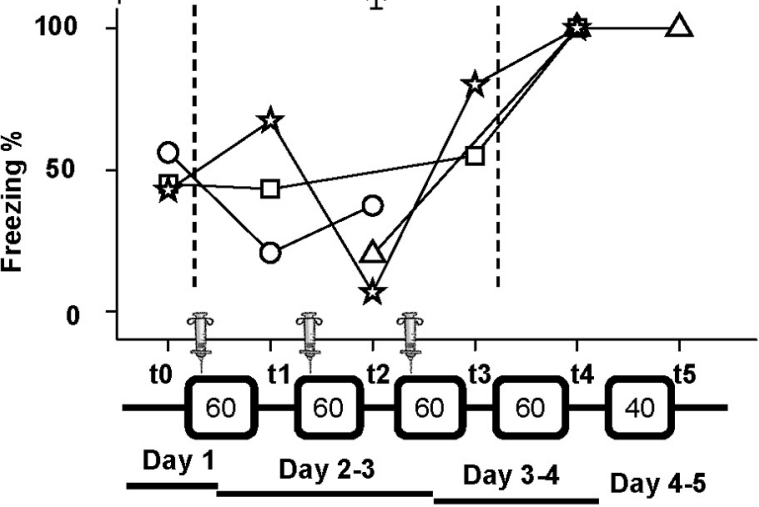

H

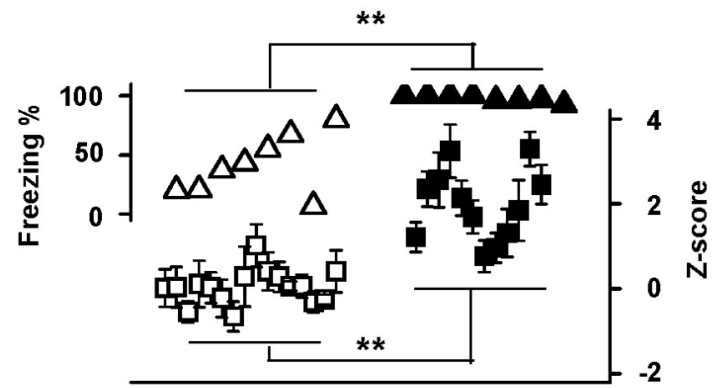

EC inactivated

EC intact

Figure 5. Unilateral entorhinal cortex inactivation blocked the encoding of the visuoauditory association. $A$, Photomicrographs of Nissl-stained brain sections of three rats showing cannula tracks. Infusions targeted the right entorhinal cortex. $\boldsymbol{B}$, Spontaneous neuronal activity in the entorhinal cortex (EC, 3 channels: $E C 1, E C 2$, and EC3) and auditory cortex (AC, 2 channels: $A C 1$ and $A C 2)$ were recorded before and $15 \mathrm{~min}$ after DNQX infusion. Recording electrodes in the entorhinal cortex were placed 1.0 to $2.0 \mathrm{~mm}$ from drug infusion cannula, and those in the auditory (Figure legend continues.) 
9.41, $p<0.001$, one-way ANOVA; ${ }^{* *} p<0.01$, post hoc). Two neurons responded to the visual stimulus during EiLR trials.

We next examined whether neuronal responses to the visual stimulus extinguished over repeated presentations of the visual stimulus (Fig. 4, see A3 for experimental timeline). Neuronal activity was monitored during the SiLR protocol from $\mathrm{t} 1$ to $\mathrm{t} 3$ and during the EiLR protocol from t4 to t5 (Fig. 4A1). Neuronal responding was suppressed after rats nose-poked the center hole and before delivery of the visual stimulus, consistent with a recent finding that engagement in a behavioral task suppresses spontaneous auditory cortex activity (Otazu et al., 2009). Neurons did not respond to the visual stimulus before conditioning ( $\mathrm{t} 0$, Fig. 4A1-2) but responded maximally at $\mathrm{t} 1$ (i.e., after 120 conditioning trials and $4 \mathrm{~d}$ of reward-retrieval training). Over the next $4 \mathrm{~d}$ $(\mathrm{t} 2-\mathrm{t} 5)$, responses to the visual stimulus gradually decreased and returned to near-pre-conditioning levels after $11 \mathrm{~d}$ and 587 trials (t5, Fig. 4A1-2). Notably, however, artificial visuoauditory memory was still detectable even after $8 \mathrm{~d}$ and 359 trials. All seven neurons responded to the visual stimulus after 200 trials, three responded to the visual stimulus after 300 trials, and none responded after 400 or more trials (Fig. $4 B$ ).

For the neurons in Figure $3(E, F, H)$, the EiLR protocol was placed after the SiLR protocol that lasted for 249-761 trials. Neuronal responses to the visual stimulus in the EiLR protocol were weaker compared to that in the SiLR protocol, partially caused by the gradual extinction effect of repeated testing trials.

\section{Entorhinal cortex inactivation impairs encoding of the visuoauditory association}

One of our main objectives was to examine the involvement of the entorhinal cortex in the encoding of an artificial visuoauditory association. To accomplish this, we pharmacologically inactivated the entorhinal cortex of the medial temporal lobe (Fig. $5 A$ ). Changes in spontaneous neuronal activity after drug infusion, which were detected by three monitoring electrodes surrounding the infusion cannula at 1.0 to $2.0 \mathrm{~mm}$ distances, indicated that inactivation was successful and spread in all directions (Fig. 5B, EC1, EC2, and EC3). In this experiment, we implanted bilateral electrode arrays, each consisting of stimulating and recording electrodes, in the auditory cortex and conditioned rats to associate a visual stimulus with electrical cortical stimulation. Drug infusion did not affect spontaneous neuronal activity of the auditory cortex, as monitored by recording electrodes ip-

$\leftarrow$

(Figure legend continued.) cortex were located at the stimulation site. C, PTSHs showing auditory cortical neuronal responses. $\boldsymbol{D}$, Raw data and raster plots of neuronal responses in left and right auditory cortices to two electrical pulses delivered to the left auditory cortex. E, Raster plots and PSTHs showing neuronal responses in right and left auditory cortices to repeated visual stimuli before conditioning ( $\mathrm{t} 0$ ), after 60 conditioning trials with the right entorhinal cortex inactivated ( $\mathrm{t} 1$ ), after 180 conditioning trials with the right entorhinal cortex inactivated $(\mathrm{t} 3)$, and after 60 additional conditioning trials with the entorhinal cortex intact (t4). One rat was eliminated from the experiment after $\mathrm{t} 2$ because no signal was detected through the recording electrodes. $F, Z$-scores of neuronal responses to the visual stimulus before $(\mathrm{t} 0)$, after conditioning with the entorhinal cortex inactivated ( $\mathrm{t} 1 \mathrm{t} \mathrm{t} 3$ ), and after further conditioning with the entorhinal cortex intact ( $t 4-\mathrm{t} 5$ ). Experimental timeline is depicted in inset below (G). DNQX or lidocaine was infused into the entorhinal cortex $15 \mathrm{~min}$ before conditioning sessions, and test trials (t1-t3) were given $4-24 \mathrm{~h}$ later, when entorhinal cortex activity had resumed. Z-scores were calculated for neuronal responses during the first $2 \mathrm{~s}$ after the visual stimulus $\left({ }^{*} p<0.05\right.$, ${ }^{* *} p<0.01$, paired $t$ test; neuronal response to the visual stimulus vs spontaneous firing). $\mathbf{G}$, Percentage time spent freezing in the $30 \mathrm{~s}$ period after each visual stimulus. Behavioral data from one of four rats at $t 0$ and $\mathrm{t} 1$ were lost. $\boldsymbol{H}$, Behavioral (\% freezing) and electrophysiological (Z-score) response to the visual stimulus with an inactivated versus intact entorhinal cortex $\left({ }^{* *} p<0.001, t\right.$ test $)$. silateral to the infusion cannula (Fig. $5 B, A C 1$ and AC2). Neuronal responses to sound confirmed the bilateral implantation of electrodes in the auditory cortex (Fig. 5C). Electrical stimulation of the left auditory cortex did not evoke neuronal responses in the right auditory cortex, indicating that the implanted sites were not strongly interconnected (Fig. 5D).

Unilateral entorhinal cortex inactivation impaired encoding of the artificial visuoauditory association as indicated by both behavioral and electrophysiological measures. After unilateral infusion of DNQX or lidocaine into the entorhinal cortex, all rats showed a behavioral deficit in fear conditioning (Fig. $5 G$ ) and a bilateral electrophysiological deficit (Fig. 5E,F), even after 120 conditioning trials ( $Z$-scores for rats \#1-4 in Fig. 5F; raster plot for rat \#3 in Fig. 5E). In two rats (\#3-4, Fig. 5F), no significant changes in $Z$-score were detected after 180 trials. By contrast, a visuoauditory association was physiologically detectable within 60 conditioning trials for all control rats (Fig. $1 F$ ).

When the same rats were exposed to 60 trials of reconditioning with an intact entorhinal cortex (i.e., when entorhinal cortex activity had resumed), the visuoauditory association was easily established (Fig. $5 E, F$ ). All rats showed significant increases in bilateral responses to the visual stimulus (Fig. 5F) and exhibited strong behavioral freezing responses, which neared $100 \%$ at $t 4$ and t5 (Fig. 5G). Freezing percentages and $Z$-scores were significantly different between inactivated and intact entorhinal cortex conditions $(p<0.001$, Fig. $5 H)$.

\section{Entorhinal cortex inactivation impairs retrieval of the visuoauditory association}

Finally, we examined the contribution of the entorhinal cortex to visuoauditory memory retrieval by inactivating the entorhinal cortex after conditioning. Unilateral entorhinal cortex inactivation impaired memory retrieval, as indicated by a behavioral and bilateral electrophysiological deficit in three out of five rats (rats \#5-7, Fig. 6A). During test sessions in which the entorhinal cortex was inactivated, these rats exhibited almost no freezing and no auditory cortical neuronal response to the visual stimulus. By contrast, during test sessions in which the entorhinal cortex was intact, the same three rats exhibited nearly $100 \%$ freezing and large bilateral auditory cortical neuronal responses to the visual stimulus (Fig. 6A). For the other two rats (rats \#1 and 4, Fig. 6B), in test sessions in which the entorhinal cortex was inactivated, auditory cortical neurons ipsilateral to the inactivated entorhinal cortex showed no response to the visual stimulus, whereas contralateral auditory cortical neurons were less affected. In test sessions in which the entorhinal cortex was intact, auditory cortical neurons responded bilaterally to the visual stimulus (Fig. 6B). These two rats exhibited nearly $100 \%$ freezing regardless of whether the entorhinal cortex was inactivated (Fig. 6B).

We observed a strong correlation between freezing and electrophysiological responses in the auditory cortex. Robust freezing was observed for all rats in which auditory cortical neurons of either hemisphere responded to the visual stimulus (i.e., positive $Z$-scores), whereas low freezing was observed for rats in which no auditory cortical neurons responded to the visual stimulus.

$Z$-scores of auditory cortex neuronal responses were significantly different between inactivated and intact entorhinal cortex conditions (Fig. 6C). There was a greater difference between conditions for ipsilateral responses $(0.06 \pm 0.19$ vs $2.1 \pm 0.55, p<$ $0.001, t$ test $)$ than for contralateral responses $(0.42 \pm 0.22$ vs $1.39 \pm 0.39, p<0.05, t$ test). There was also a difference in freezing between the two conditions, but this was not statistically significant $(p=0.107)$. 

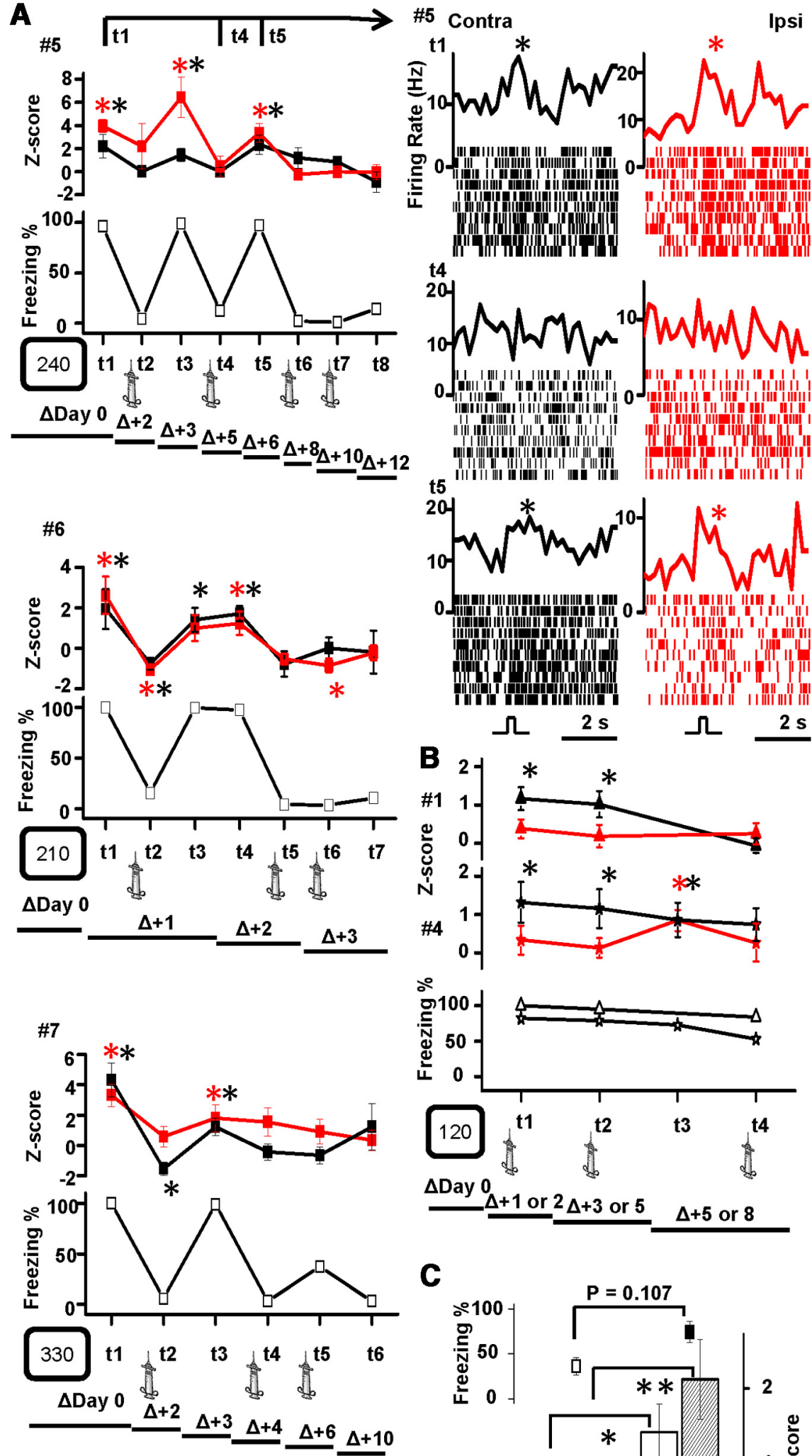

C

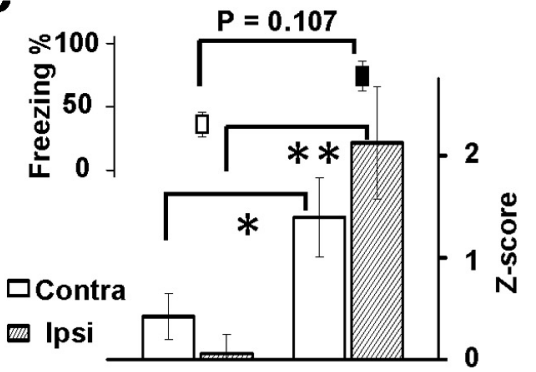

EC inactivated EC intact

Figure 6. Unilateral entorhinal cortex inactivation impaired retrieval of the visuoauditory association. $A, Z$-scores of neuronal responses to the visual stimulus and percentage time spent freezing at different time points indicated in the insets below each graph. On day 0, baseline data were collected and conditioning occurred. On infusion days, data were collected 15 min after infusion of DNQX into the entorhinal cortex. Z-scores for rats \#5 (top), 6 (middle), and 7 (bottom) were calculated over the first 0.5 ,

\section{Discussion}

Our main finding was that an artificial cross-modal association between a visual stimulus and electrical auditory cortex stimulation can be established within $20-30$ conditioning trials. Establishment of this association was monitored using a combined stimulating and recording electrode array. Unilateral inactivation of the entorhinal cortex completely blocked the visuoauditory association, although the association was easily established in the same rats with an intact entorhinal cortex. Unilateral entorhinal cortex inactivation also affected the retrieval of the visuoauditory association in the early days of its consolidation. Furthermore, entorhinal cortex inactivation resulted in a reduced freezing response to the conditioned stimulus.

Classical conditioning induces specific changes in the frequency receptive fields of single neurons in the auditory cortex (Diamond and Weinberger, 1986; Bakin and Weinberger, 1990) that are observable after five conditioning trials and stabilize after 30 trials (Edeline et al., 1993). Moreover, plasticity in the auditory cortex can be induced after both classical and instrumental conditioning (Recanzone et al., 1993; Weinberger, 2007; Scheich and Ohl, 2011), including detection, discrimination, and categorization learning tasks (Weinberger, 2007; Scheich and Ohl, 2011). Previous studies suggest that experience with one sensory modality can influence the activity of cortical areas associated with other sensory modalities. For instance, in humans, an auditory stimulus can activate the visual cortex if it is paired with a visual stimulus during conditioning (McIntosh and GonzalezLima, 1998; McIntosh et al., 1998; Zangenehpour and Zatorre, 2010), the

1.0 , and $0.5 s$ after the visual stimulus, respectively. The right panel shows PSTHs and raster plots of bilateral neuronal responses to repeated visual stimuli in the auditory cortex in rat $\# 5$ at $\mathrm{t} 1$, $\mathrm{t} 4$, and $\mathrm{t} 5$ ( $^{*} p<0.05$, paired $t$ test; neuronal response to the visual stimulus vs spontaneous firing). The number in the box above " $\Delta$ Day 0 " indicates the number of trials used to establish the associative memory for each rat. $\boldsymbol{B}, \mathbf{Z}$-scores of neuronal responses to the visual stimulus and percentage time spent freezing in rats \#1 and 4 at different time points as shown in the inset below the graph. On infusion days, data were acquired $15 \mathrm{~min}$ after infusion. Z-scores for rats \#1 and 4 were calculated over the first $2 \mathrm{~s}$ after the visual stimulus. Three rats successfully completed this experiment. C, Behavioral (\% freezing) and electrophysiological (Z-score) response with an inactivated versus intact entorhinal cortex $\left({ }^{*} p<0.05\right.$, ${ }^{* *} p<0.001, t$ test). Mean Z-scores for hemispheres ipsilateral and contralateral to the drug-infused entorhinal cortex were plotted separately. 
auditory cortex is activated by silent lip-reading and visual stimuli (Calvert et al., 1997; Brosch et al., 2005, 2011), and the visual cortex is activated by auditory stimuli (Crottaz-Herbette et al., 2004; Martuzzi et al., 2007). In trained monkeys, somatosensory neurons respond to auditory and/or visual stimuli (Zhou and Fuster, 2000; Brosch et al., 2011), prefrontal cortical neurons respond to both auditory and visual stimuli (Watanabe, 1992; Fuster et al., 2000; Sugihara et al., 2006; Romanski, 2007), and exposure to an eight-degree mismatch between auditory and visual spatial inputs shifts the perception of auditory space (Recanzone, 1998). In the present study, we explored how two physically separate sensory cortical areas engage to establish an artificial association following brief behavioral training in rats. We show for the first time that auditory cortex neurons can respond to a purely visual stimulus following brief exposure to bimodal audiovisual stimuli (Meyer et al., 2007; Zangenehpour and Zatorre, 2010).

Recent studies show that cross-modal associations and emotional memories are likely to be stored in the association auditory cortex (Meyer et al., 2007; Sacco and Sacchetti, 2010). We confirmed that the association auditory cortex can be linked cross-modally to a visual stimulus (Fig. 3B). However, we cannot exclude the possible involvement of the primary auditory cortex in the cross-modal association, as the association was established in six rats without distinction between primary and association cortices (see also Weinberger, 2004). The primary auditory cortex is known to be engaged in the association of two auditory tones (Weinberger, 2007). Moreover, auditory and visual cortices are interconnected (Falchier et al., 2002; Rockland and Ojima, 2003; Bizley et al., 2007) and further connected to the medial temporal lobe (Witter and Groenewegen, 1986; Insausti et al., 1997; Brown and Aggleton, 2001; Mohedano-Moriano et al., 2007), and the auditory cortex is reciprocally connected to the entorhinal cortex (Budinger et al., 2000, 2008). These anatomical results provide the structural foundation for participation of the medial temporal lobe in the visuoauditory associative memory establishment.

The protocol used in the present study opens new avenues for future investigations of learning and memory. For example, using simultaneous recordings of different brain regions in behaving rats undergoing conditioning, the involvement of the amygdala in the learning process and the mechanisms by which the dorsal thalamus communicates the association of visual and auditory signals to the medial temporal lobe (including the amygdala) can be determined (Romanski and LeDoux, 1992). Auditory fear conditioning, which is disrupted by amygdala lesions (Wilensky et al., 1999), is a powerful protocol for characterizing the mechanisms of learning and memory (Johansen et al., 2011). Furthermore, fear-based associative memory protocols can be used to facilitate the encoding of memory in the hippocampus and cortex.

A critical aspect of our study is the use of the reward retrieval task to confirm that rats recalled the association between the visual stimulus and electrical cortical stimulation. Their ability to successfully retrieve the reward immediately after cortical stimulation was replaced with the visual stimulus, which is indicative of the strength of the association between the two stimuli, as until that point in time the visual stimulus had never signaled reward availability. Electrophysiological recordings during reward retrieval further showed that auditory cortical neurons responded to the visual stimulus. It is interesting to note that the visuoauditory association established using a fear conditioning protocol could be retrieved in a reward-based operant conditioning protocol. However, fear conditioning was not necessary for the establishment of the visuoauditory associative memory. Furthermore, although these experiments were not de- signed to examine memory extinction in detail, changes in lighttriggered auditory cortex neuronal responses provide clues about the time course of extinction.

According to the hippocampus-neocortex-transfer theory, recent memories primarily activate the hippocampus, whereas remote memories primarily activate the neocortex (Alvarez and Squire, 1994; Wiltgen et al., 2004). A possible explanation for the slow onset of cortical involvement in memory is that new memories must be linked to existing memories within the cortex via a gradual interleaving process to avoid damaging the existing memories (McClelland et al., 1995). Our finding that artificial visuoauditory memory was established in the auditory cortex within $10 \mathrm{~min}$ of conditioning indicates that this memory is quickly stored in the cortex, which supports a revised theory in which the neocortex is activated by and involved in the storage of recent as well as remote memories [McClelland et al. (1995), their Fig. 2]. Our finding that the establishment of artificial visuoauditory memory in the rat auditory cortex depends on the entorhinal cortex substantiates findings in humans that new and enduring associative memories cannot be established without medial temporal lobe involvement (Scoville and Milner, 1957; Corkin, 1984; Squire et al., 2001).

Most connections between the hippocampus and cortex consist of entorhinal-perirhinal-cortical and entorhinal-cortical connections (Swanson and Köhler, 1986; Brown and Aggleton, 2001). Hence, the entorhinal cortex is a key intermediary structure. We found that unilateral inactivation of the entorhinal cortex prevented the establishment of bilateral artificial visuoauditory associations in the auditory cortex. This finding is consistent with previous studies reporting that bilateral inactivation of the perirhinal cortex disrupts the encoding and retrieval of object recognition memory (Winters and Bussey, 2005) and that entorhinal cortex stimulation enhances memory (Suthana et al., 2012). Also, we found that the entorhinal cortex is involved in the retrieval of established visuoauditory associations, at least shortly after memory encoding. Furthermore, electrophysiological measures of memory retrieval correlated with behavioral freezing to the conditioned stimulus. Our findings support previous observations that entorhinal cortical neurons project bilaterally to the neocortex (Swanson and Köhler, 1986). Within days of the establishment of an artificial visuoauditory association, unilateral entorhinal cortex inactivation completely suppressed memory retrieval in the ipsilateral auditory cortex and partially suppressed memory retrieval in the contralateral auditory cortex. Our results suggest that the medial temporal lobe is necessary for the encoding and involved in the retrieval (albeit to a lesser extent) of an artificial visuoauditory association in the auditory cortex early during memory consolidation.

\section{References}

Alvarez P, Squire LR (1994) Memory consolidation and the medial temporal lobe: a simple network model. Proc Natl Acad Sci U S A 91:7041-7045. CrossRef Medline

Bakin JS, Weinberger NM (1990) Classical conditioning induces CSspecific receptive field plasticity in the auditory cortex of the guinea pig. Brain Res 536:271-286. CrossRef Medline

Bizley JK, Nodal FR, Bajo VM, Nelken I, King AJ (2007) Physiological and anatomical evidence for multisensory interactions in auditory cortex. Cereb Cortex 17:2172-2189. Medline

Brosch M, Selezneva E, Scheich H (2005) Nonauditory events of a behavioral procedure activate auditory cortex of highly trained monkeys. J Neurosci 25:6797-6806. CrossRef Medline

Brosch M, Selezneva E, Scheich H (2011) Formation of associations in auditory cortex by slow changes of tonic firing. Hear Res 271:66-73. CrossRef Medline

Brown MW, Aggleton JP (2001) Recognition memory: what are the roles of 
the perirhinal cortex and hippocampus? Nat Rev Neurosci 2:51-61. CrossRef Medline

Budinger E, Heil P, Scheich H (2000) Functional organization of auditory cortex in the Mongolian gerbil (Meriones unguiculatus). III. Anatomical subdivisions and corticocortical connections. Eur J Neurosci 12:2425-2451. CrossRef Medline

Budinger E, Laszcz A, Lison H, Scheich H, Ohl FW (2008) Non-sensory cortical and subcortical connections of the primary auditory cortex in Mongolian gerbils: Bottom-up and top-down processing of neuronal information via field AI. Brain Res 1220:2-32. CrossRef Medline

Buonomano DV (2003) Timing of neural responses in cortical organotypic slices. Proc Natl Acad Sci U S A 100:4897-4902. CrossRef Medline

Cahill L, Ohl F, Scheich H (1996) Alteration of auditory cortex activity with visual stimulus through conditioning: a 2-deoxyglucose analysis. Neurobiol Learn Memory 65:213-222. CrossRef

Calvert GA, Bullmore ET, Brammer MJ, Campbell R, Williams SC, McGuire PK, Woodruff PW, Iversen SD, David AS (1997) Activation of auditory cortex during silent lipreading. Science 276:593-596. CrossRef Medline

Corkin S (1984) Lasting consequences of bilateral medial temporal lobectomy: Clinical course and experimental findings in H.M. Semin Neurol 4:249-259. CrossRef

Crottaz-Herbette S, Anagnoson RT, Menon V (2004) Modality effects in verbal working memory: differential prefrontal and parietal responses to auditory and visual stimuli. Neuroimage 21:340-351. CrossRef Medline

Diamond DM, Weinberger NM (1986) Classical conditioning rapidly induces specific changes in frequency receptive fields of single neurons in secondary and ventral ectosylvian auditory cortical fields. Brain Res 372:357-360. CrossRef Medline

Edeline JM, Pham P, Weinberger NM (1993) Rapid development of learning-induced receptive field plasticity in the auditory cortex. Behav Neurosci 107:539-551. CrossRef Medline

Falchier A, Clavagnier S, Barone P, Kennedy H (2002) Anatomical evidence of multimodal integration in primate striate cortex. J Neurosci 22:57495759. Medline

Fuster JM, Bodner M, Kroger JK (2000) Cross-modal and cross-temporal association in neurons of frontal cortex. Nature 405:347-351. CrossRef Medline

Graham KS, Hodges JR (1997) Differentiating the roles of the hippocampal complex and the neocortex in long-term memory storage: evidence from the study of semantic dementia and Alzheimer's disease. Neuropsychology 11:77-89. CrossRef Medline

He J (1997) Modulatory effects of regional cortical activation on the onset responses of the cat medial geniculate neurons. J Neurophysiol 77:896-908. Medline

Insausti R, Herrero MT, Witter MP (1997) Entorhinal cortex of the rat: cytoarchitectonic subdivisions and the origin and distribution of cortical efferents. Hippocampus 7:146-183. Medline

Johansen JP, Cain CK, Ostroff LE, LeDoux JE (2011) Molecular mechanisms of fear learning and memory. Cell 147:509-524. CrossRef Medline

Johnson HA, Goel A, Buonomano DV (2010) Neural dynamics of in vitro cortical networks reflects experienced temporal patterns. Nat Neurosci 13:917-919. CrossRef Medline

Kayser C, Petkov CI, Logothetis NK (2008) Visual modulation of neurons in auditory cortex. Cereb Cortex 18:1560-1574. Medline

Lesburguères E, Gobbo OL, Alaux-Cantin S, Hambucken A, Trifilieff P, Bontempi B (2011) Early tagging of cortical networks is required for the formation of enduring associative memory. Science 331:924-928. CrossRef Medline

Martuzzi R, Murray MM, Michel CM, Thiran JP, Maeder PP, Clarke S, Meuli RA (2007) Multisensory interactions within human primary cortices revealed by BOLD dynamics. Cereb Cortex 17:1672-1679. Medline

McClelland JL, McNaughton BL, O’Reilly RC (1995) Why there are complementary learning systems in the hippocampus and neocortex: insights from the successes and failures of connectionist models of learning and memory. Psychol Rev 102:419-457. CrossRef Medline

McIntosh AR, Gonzalez-Lima F (1998) Large-scale functional connectivity in associative learning: interrelations of the rat auditory, visual, and limbic systems. J Neurophysiol 80:3148-3162. Medline

McIntosh AR, Cabeza RE, Lobaugh NJ (1998) Analysis of neural interactions explains the activation of occipital cortex by an auditory stimulus. J Neurophysiol 80:2790-2796. Medline

Meyer M, Baumann S, Marchina S, Jancke L (2007) Hemodynamic re- sponses in human multisensory and auditory association cortex to purely visual stimulation. BMC Neurosci 8:14. CrossRef Medline

Mohedano-Moriano A, Pro-Sistiaga P, Arroyo-Jimenez MM, Artacho-Pérula E, Insausti AM, Marcos P, Cebada-Sánchez S, Martínez-Ruiz J, Muñoz M, Blaizot X, Martinez-Marcos A, Amaral DG, Insausti R (2007) Topographical and laminar distribution of cortical input to the monkey entorhinal cortex. J Anat 211:250-260. CrossRef Medline

Otazu GH, Tai LH, Yang Y, Zador AM (2009) Engaging in an auditory task suppresses responses in auditory cortex. Nat Neurosci 12:646-654. CrossRef Medline

Recanzone GH (1998) Rapidly induced auditory plasticity: the ventriloquism aftereffect. Proc Natl Acad Sci U S A 95:869-875. CrossRef Medline

Recanzone GH, Schreiner CE, Merzenich MM (1993) Plasticity in the frequency representation of primary auditory cortex following discrimination training in adult owl monkeys. J Neurosci 13:87-103. Medline

Rockland KS, Ojima H (2003) Multisensory convergence in calcarine visual areas in macaque monkey. Int J Psychophysiol 50:19-26. CrossRef Medline

Romanski LM (2007) Representation and integration of auditory and visual stimuli in the primate ventral lateral prefrontal cortex. Cereb Cortex 17:i6169. CrossRef Medline

Romanski LM, LeDoux JE (1992) Equipotentiality of thalamo-amygdala and thalamo-cortico-amygdala circuits in auditory fear conditioning. J Neurosci 12:4501-4509. Medline

Sacco T, Sacchetti B (2010) Role of secondary sensory cortices in emotional memory storage and retrieval in rats. Science 329:649-656. CrossRef Medline

Scheich H, Ohl FW (2011) A semantic concept of auditory cortex function and learning. In: Auditory cortex (Winer JA, CE Schreiner, eds), pp 369 387. New York: Springer.

Scheich H, Brechmann A, Brosch M, Budinger E, Ohl FW, Selezneva E, Stark H, Tischmeyer W, Wetzel W (2011) Behavioral semantics of learning and crossmodal processing in auditory cortex: the semantic processor concept. Hear Res 271:3-15. CrossRef Medline

Scoville WB, Milner B (1957) Loss of recent memory after bilateral hippocampal lesions. J Neurol Neurosurg Psychiatry 20:11-21. CrossRef Medline

Squire LR, Clark RE, Knowlton BJ (2001) Retrograde amnesia. Hippocampus 11:50-55. CrossRef Medline

Sugihara T, Diltz MD, Averbeck BB, Romanski LM (2006) Integration of auditory and visual communication information in the primate ventrolateral prefrontal cortex. J Neurosci 26:11138-11147. CrossRef Medline

Suthana N, Haneef Z, Stern J, Mukamel R, Behnke E, Knowlton B, Fried I (2012) Memory Enhancement and Deep-Brain Stimulation of the Entorhinal Area. N Engl J Med 366:502-510. CrossRef Medline

Swanson LW, Köhler C (1986) Anatomical evidence for direct projections from the entorhinal area to the entire cortical mantle in the rat. J Neurosci 6:3010-3023. Medline

Teng E, Squire LR (1999) Memory for places learned long ago is intact after hippocampal damage. Nature 400:675-677. CrossRef Medline

Wang SH, Teixeira CM, Wheeler AL, Frankland PW (2009) The precision of remote context memories does not require the hippocampus. Nat Neurosci 12:253-255. CrossRef Medline

Watanabe M (1992) Frontal units of the monkey coding the associative significance of visual and auditory stimuli. Exp Brain Res 89:233-247. Medline

Weinberger NM (2004) Specific long-term memory traces in primary auditory cortex. Nat Rev Neurosci 5:279-290. CrossRef Medline

Weinberger NM (2007) Auditory associative memory and representational plasticity in the primary auditory cortex. Hear Res 229:54-68. CrossRef Medline

Wilensky AE, Schafe GE, LeDoux JE (1999) Functional inactivation of the amygdala before but not after auditory fear conditioning prevents memory formation. J Neurosci 19:RC48. Medline

Wiltgen BJ, Brown RA, Talton LE, Silva AJ (2004) New circuits for old memories: the role of the neocortex in consolidation. Neuron 44:101108. CrossRef Medline

Winters BD, Bussey TJ (2005) Transient inactivation of perirhinal cortex disrupts encoding, retrieval, and consolidation of object recognition memory. J Neurosci 25:52-61. CrossRef Medline

Witter MP, Groenewegen HJ (1986) Connections of the parahippocampal 
cortex in the cat. III. Cortical and thalamic efferents. J Comp Neurol 252:1-31. CrossRef Medline

Yu XJ, Xu XX, He S, He J (2009) Change detection by thalamic reticular neurons. Nat Neurosci 12:1165-1170. CrossRef Medline

Yu YQ, Xiong Y, Chan YS, He J (2004) Corticofugal gating of auditory information in the thalamus: an in vivo intracellular recording study. J Neurosci 24:3060-3069. CrossRef Medline
Zangenehpour S, Zatorre RJ (2010) Crossmodal recruitment of primary visual cortex following brief exposure to bimodal audiovisual stimuli. Neuropsychologia 48:591-600. CrossRef Medline

Zhou YD, Fuster JM (2000) Visuo-tactile cross-modal associations in cortical somatosensory cells. Proc Natl Acad Sci U S A 97:9777-9782. CrossRef Medline 